\title{
Umbilical hernia, epigastric hernia and diastasis recti: an open discussion
}

\author{
G. Campanelli ${ }^{1}$
}

Published online: 2 June 2021

(c) The Author(s), under exclusive licence to Springer-Verlag France SAS, part of Springer Nature 2021

Some situations in routine general surgical practice are so clear cut that no debate or discussion is necessary and the surgeon can proceed without too much hesitation.

Unfortunately, when it comes to abdominal wall hernia surgery, such situations are much less frequent because, in this field, there is often a lack of clear, reproducible and reliable clinical evidence.

And this is despite the fact that international societies and experts' groups the world over have poured considerable energy into trying to provide general surgeons with guidance on the most reasonable choices to make when faced with the various abdominal wall disorders that crop up in daily practice.

I am not talking about abdominal wall disasters, complications or difficult situations requiring super-specialist knowledge and expertise-I have the impression that these nowadays tend to be treated in dedicated hernia centers-, but rather routine surgeries for problems such as umbilical and/or epigastric hernia, with or without associated diastasis recti. Let us not forget that these hernias are the second most frequent type dealt with in theaters around the world.

Despite the great work done by researchers and hernia societies, and the publication of guidelines, trials and observational studies, the quality of the evidence remains poor, and the debate continues to be marred by numerous sources of bias as well as open questions.

First of all, the presence of diastasis recti-leaving aside the difficulties in arriving at a shared classification of this condition-is a watershed point in the decision algorithm. For this reason, it is always crucial to evaluate, during a thorough CT scan, the inner-recti distance, to verify that it is (more or less) normal, both in rest and in dynamic conditions.
Second, the size of the defect and the possible simultaneous presence of umbilical and epigastric (sometimes multi-epigastric) defects, as well as the patient's anatomical characteristics and BMI are other aspects that, together, determine a set of choices that the surgeon must consider carefully in order to identify, in line with a tailored approach, the one best suited to the patient in question.

There is partial consensus on simple (non-absorbable) sutures of small (i.e., maximum $1.5-2 \mathrm{~cm}$ ) umbilical or single small epigastric hernias, provided there is no diastasis and the patient has a physiological inter-recti distance; as well as there is partial consensus on complete prosthetic repair retroxifo Douglas line between the two lateral margins of rectus muscles in the same cases, although with a real diastasis recti it is mandatory to perform complete plication and reconstruction of the linea alba using a mini/less open, lap (IPOM, e-TEP) or robotic technique.

The scientific community seems to be pretty much in agreement over these two situations, but what about the rest?

We always opt for prosthetic repair in cases with larger defects (using open, lap or robotic techniques, regardless of whether there is a diastasis, but what should be the extension of the repair? Should it be limited to the defect or a bit more extensive? Or should we opt for complete repair of the wall (as described above)? What about larger inter-recti distances, like those encountered in advanced diastasis, where re-approximation of the linea alba without strong tension is impossible? And in these cases, is component separation justified? And, if so, which kind?

All these are questions and doubts that still need to be discussed and analyzed. We, therefore, urge you to contribute to this crucial debate, by continuing to share your experiences with our journal.

G. Campanelli

hernia.editorialoffice@gmail.com

1 University of Insubria, Gruppo Ospedaliero San Donato, Milan, Italy 


\section{Declarations}

Publisher's Note Springer Nature remains neutral with regard to jurisdictional claims in published maps and institutional affiliations.

Conflict of interest None.

Ethical approval Not applicable.

Human and animal rights Not applicable.

Informed consent Not applicable. 